\title{
An Enhanced Technique for Recording and Analysis of Electrogastrogram using Active Electrodes
}

\author{
Prof. G.Gopu MTech \\ Research Scholar, Department of Electrical and Electronics Engineering, PSG College of Technology, \\ Coimbatore-04, Tamilnadu, India \\ Dr. R.Neelaveni ME, PhD \\ Assistant Professor, Department of Electrical and Electronics Engineering, PSG College of Technology, \\ Coimbatore-04, Tamilnadu, India
}

Dr. K.Porkumaran ME, PhD

Professor and Head, Department of Biomedical Engineering, Sri Ramakrishna Engineering College, Coimbatore-22,Tamilnadu, India.

Dr. M.G.Shekar MS, DNB, MRCS

General and Laparoscopic Surgeon, Stanley Medical College and Hospital, Chennai-01, Tamilnadu, India E-mail address: Dr. G. Gopu - gopugovindasamy@gmail.com

Sri Lanka Journal of Bio-Medical Informatics 2010;1(1):21-27

DOI: 10.4038/sljbmi.v1i1.1482

\begin{abstract}
Electrogastrogram (EGG) is obtained with the help of Active Electrode (AE) from the human being cutaneously. It is a non-invasive, easy procedure that is tried as a preliminary investigation to assess the need for endoscopy. Active electrode setup is proposed in this paper to acquire the myoelectrical activity of the stomach as it is of higher sensitivity in acquiring the EGG signals when compared to passive electrodes. Active electrode based acquisition is most reliable as signal loss is prevented. Interference is prevented completely by using appropriate filters and by using Electromagnetic Interference (EMI) shield. EGG recorded in more than hundred and fifty human being includes the normal individual and patients with digestive system disorders such us nausea, dyspepsia, vomiting, peptic ulcer, etc. A database is created for the analysis. The change in frequency and power for disorders in patients compared to normal individuals form the power spectrum plot. Wavelet Transform analysis includes Continuous Wavelet Transform (CWT) to analyze the signal for the diagnosis of various gastric disorders as mentioned above. In wavelet analysis, the data of a patient or normal individual is considered. The signal is reconstructed with a data obtained through a data scope. This input signal undergoes Principle Component Analysis (PCA) as a preprocessing manoeuvre and then the interference and noise in the EGG signals are removed to obtain de-noised signal. The denoised EGG signal is plotted for power spectral density estimation with Welch power spectral Density Estimation. As a result of the proposed method the power variation is found to be $40-52 \mathrm{~dB}$ and frequency is detected as $6.0-8.5 \mathrm{cpm}$ in preprandial and $1.5-2.5 \mathrm{cpm}$ in postprandial for ulcer patients, $4-5 \mathrm{cpm}$ in preprandial $1.5-2.8 \mathrm{cpm}$ in postprandial for dyspepsia patients and $3.0-3.15 \mathrm{cpm}$ for normal individuals.
\end{abstract}

Keywords : Active Electrode Electrogastrogram, Electromagnetic Interference, Preprandial, Postprandial, CWT

\section{Introduction}

Gastric Electrical Activity (GEA) due to stomach motility measured cutaneously using the surface electrodes $(\mathrm{Ag} / \mathrm{Agcl})$ is called Electrogastrography ${ }^{(1,2)}$. EGG includes the fundamental frequency such us electrical control activity of the stomach (ECA) and the electrical response activity (ERA). Almost 75 years ago W.C Alvarez published about the EGG but the first recording of gastro electrical activity was performed in dogs by C.F. Code and J.A.Marleh in the year $1974^{(3)}$ and in human in $1978^{(4)}$ by R.L.Telander et al. But the progress in this field has been very slow, especially compared with other cutaneous electrophysiological measurements, such as Electrocardiography, because of its difficulty in data acquisition, lack of understanding, etc. Due to the advances in quantitative analysis of the EGG, more and more physicians and biomedical researchers are interested in this field. The abnormalities in EGG arises due to recurrent nausea, vomiting, dyspepsia, stomach ulcers, cyclic vomiting 
syndrome, tachygastria, bradygastria, etc which results in improper digestion of food by the stomach. If the EGG is abnormal, it confirms that the problem probably is with the stomach's muscles or the nerves that control the muscles. This paper mainly deals with the denoising of EGG using wavelets Daubechies wavelet Transform. The denoising of the EGG by db4 wavelet is found good compare to Haar and other wavelets. The EGG of preprandial and postprandial for a subjects with Bradygastria and with Tachygastria is obtained by the Continuous Wavelet Transform [CWT]. The denoised EGG signal's frequency and power in $\mathrm{db}$ is obtained with Welch power Spectral Density Estimation. More than 150 subjects with gastric disorders, normal were studied for the investigation of the digestive system disorders as mentioned above.

\section{Materials and Methods}

\section{Electrogastrogram}

An EGG is similar to an electrocardiogram of the heart. It is a recording of the electrical signals that travel through the muscles of the stomach and control the muscle's contraction $(14,15,16)$. EGG is used when there is a suspicion that the muscles of the stomach or the nerves controlling the muscles are not working normally. EGG is done by placing the electrode cutaneously over the stomach and the electrical signals coming from the stomach muscles are sensed by the electrode and recorded on a computer for analysis by lying the patient quietly. In normal individuals the EGG is a regular electrical rhythm generated by the muscles of the stomach and the power (voltage) of the electrical current increases after a meal. In patients with abnormalities of the muscles or nerves of the stomach, the rhythm often is irregular or there is no post-meal increase in electric power. EGG will not have any side effects and it is a painless study. The EGG wave pattern before and after denoising is shown in Figure 1.

Figure 1. A and B are EGG waveform before filtering and after filtering respectively.

A

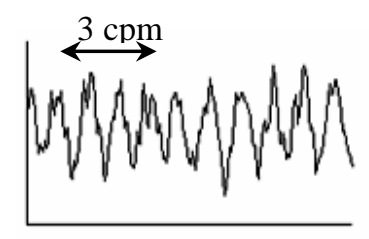

B

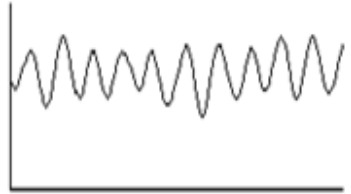

\section{Proposed Recording Setup}

In this proposed recoding setup the active electrodes are used to acquire the electrical signals directly from the stomach, which are used to buffer the signals and also provide impedance matching. The active electrodes are well protected within a shield to reduce electromagnetic interference. EGG from the active electrode undergoes signal conditioning in a SCU unit, which include instrumentation amplifier (IA) and filter. IA is high input impedance and high CMRR about $100 \mathrm{db}$ with variable gain of 1 to 10000. The noise due to respiratory, cardiac and other bio-signals along with motion artifacts is also acquired with EGG. This is eliminated in the filter section and the frequency range from 1 to $10 \mathrm{cpm}$ of EGG is viewed in the digital storage scope (DSO).The EGG given to data scope to convert the analog form into digital form to store as database in the PC via RS 232 serial interface for analysis and classification to assist the physician in the investigation of digestive system disorder. The general block diagram for the above said proposed recoding setup is shown in Figure 2. 
Figure 2. General Block Diagram

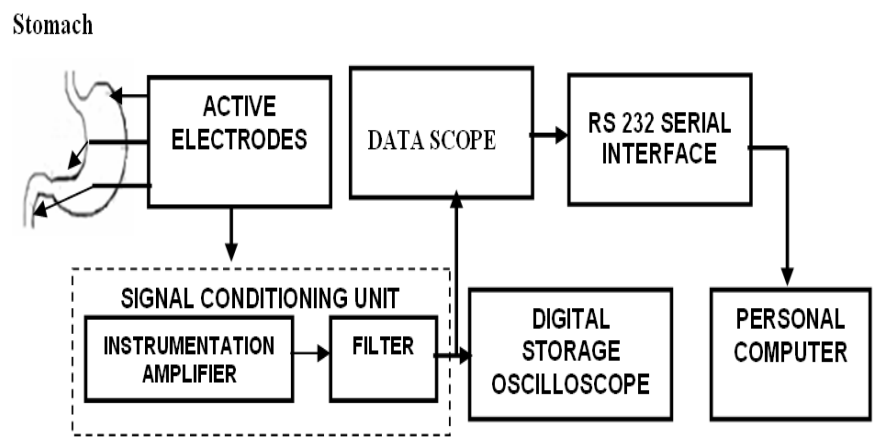

\section{Active Electrode Position}

The electrical signals are generally produced in the mid-corpus of the stomach where the electrical activity takes place. The positioning of the active electrodes for tapping these signals is as shown in Figure 3(A) and the real time acquisition with $\mathrm{AE}$ is shown in Figure 3(B). Two electrodes A and B are placed in the fundus and the mid corpus of the stomach. The third electrode $\mathrm{C}$ is placed as ground at the end of the stomach region for patient safety $(5,6,7)$.

Figure 3. Active Electrodes Positioning for Recording EGG

A

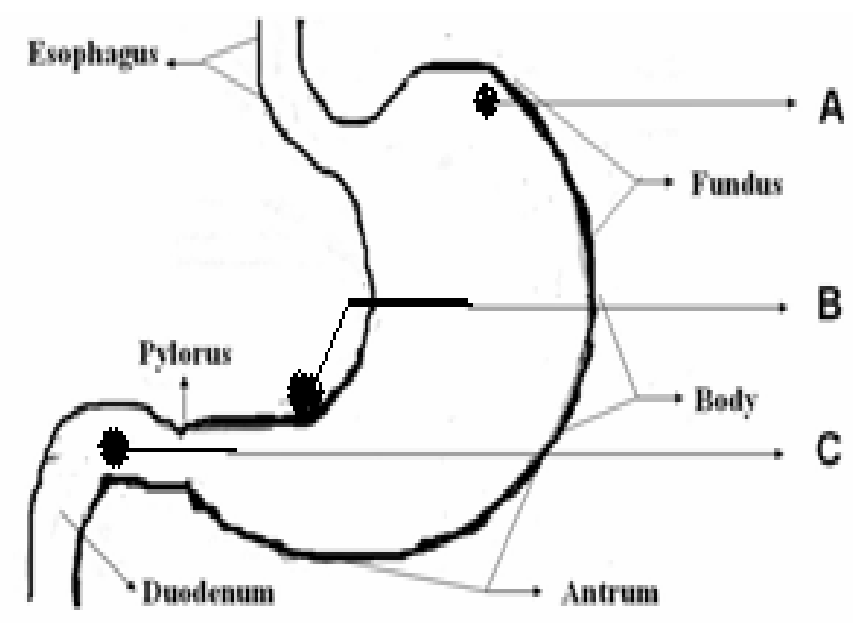

B

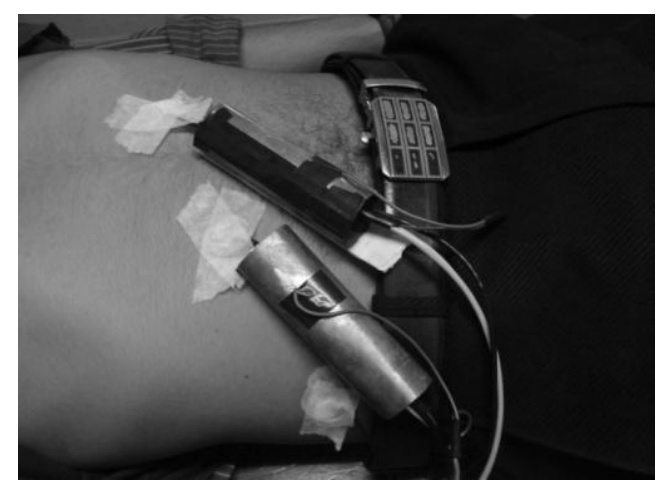

\section{Subjects}

Subjects consecutively attending a hospital outpatient's gastroenterology clinic and students with disorders were studied for this investigation at biomedical department of our institution and gastroenterology department of a reputed hospital. More than hundred subjects including patients and normal subjects, both male and female, of different age groups participated in this recording ${ }^{(8,10)}$. The patients included 40 with dyspepsia, 45 with stomach ulcers and 25 with nausea, 25 with bradygastria and 20 with tachygastria. The acquisition of data started four years ago and is being continued. The various categories of subjects are listed in Table 1. 
The electrodes are positioned as described above. The output of the electrode is given to the instrumentation amplifier of the recording setup. The amplified signal undergoes preprocessing and then the data are acquired via data scope and the same is stored in a database in the computer for further analysis. In this paper, EGG data are reconstructed as an original EGG signal and the same is included in the PCA as a preprocessing step to get actual EGG signals. Denoising of the half an hour recorded EGG signal is performed with wavelet denoising technique to find the frequency and power spectrum. Figure 4 represents the denoising of an EGG signal with Haar wavelet and Figure 5 represents the denoising of a EGG signal with db4 wavelet. As a finding the denoising by db4 is better than Haar wavelet.

Table 1. Age and Sex distribution of patient groups

\begin{tabular}{|c|c|c|c|}
\hline Disorders & $\begin{array}{c}\text { Mean Age } \\
\text { (years) }\end{array}$ & $\begin{array}{c}\text { Mal } \\
\text { e }\end{array}$ & Female \\
\hline Dyspepsia (n=40) & 38 & 24 & 16 \\
\hline $\begin{array}{c}\text { Stomach Ulcer } \\
(\mathbf{n = 4 5 )}\end{array}$ & 34 & 26 & 19 \\
\hline Nausea (n=25) & 45 & 25 & 20 \\
\hline Bradygastria (n=25) & 28 & 15 & 10 \\
\hline Tachygastria (n=20) & 36 & 12 & 08 \\
\hline
\end{tabular}

Figure 4. Denoising of EGG signal with Haar wavelet transforms
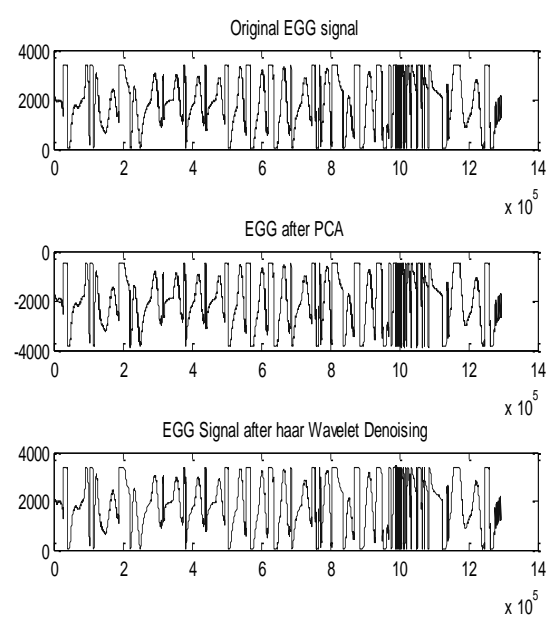

Figure 5. Denoising of EGG signal with db4 wavelet transforms.
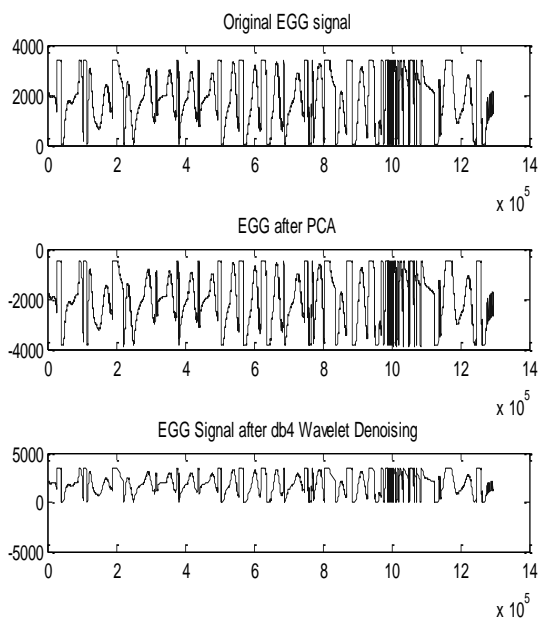

\section{Results and Discussion}

The denoising of the EGG signal using wavelet transform increases the reduction of noise signal which is under or above EGG frequency limit. From the database the data analyzed for detecting frequency and power of the EGG signal of the normal subjects and the patients with digestive system disorders after wavelet denoising using Welch power spectral Density Estimation as shown in Figure 6, which shows the range of frequencies and power for the dyspepsia patient with frequency $0.02344 \mathrm{~Hz}$ and power $48.42 \mathrm{db}$ in postprandial condition. Like this the frequency and power can be found for the other digestive disorder subjects such as 6.0-8.5 cpm for ulcer and dyspepsia patients and 3.0-3.15 cpm for normal individual. In this study, we have used the EGG setup to record myoelectrical activity for the patients 
suffering from dyspepsia, stomach ulcer, tachygastria, bradygastria and normal subjects for both preprandial and postprandial condition ${ }^{(9,11)}$. Figure 7 and Figure 8 show the $\mathrm{CWT}^{(17)}$ for a normal person in preprandial condition with 3 peaks and postprandial condition with increased gastric activity. The condition of bradygastria and tachygastria is represented with CWT in figure 9 and figure 10 respectively. It is observed that the slow frequency wave for bradygastria and fast frequency wave for tachygastria. It was observed that there is a power raise postprandial in normal individuals and no power raise postprandial in patients with dyspepsia (Figure 11).

Figure 6. Welch power spectral Density Estimation for disorder subjects

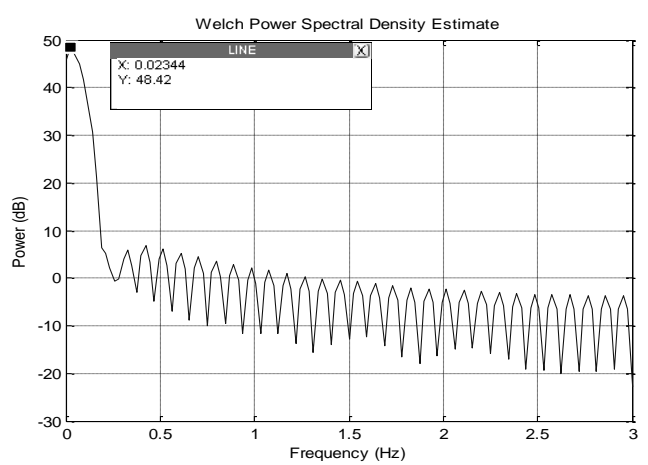

Figure 8. CWT of postprandial EGG

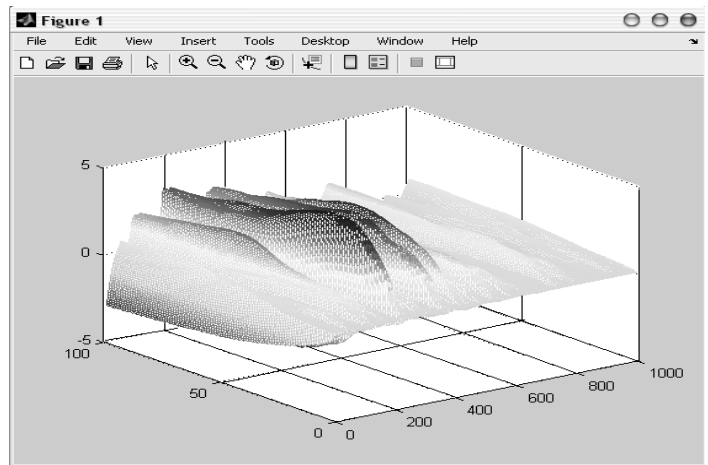

Figure 7. CWT of preprandial EGG

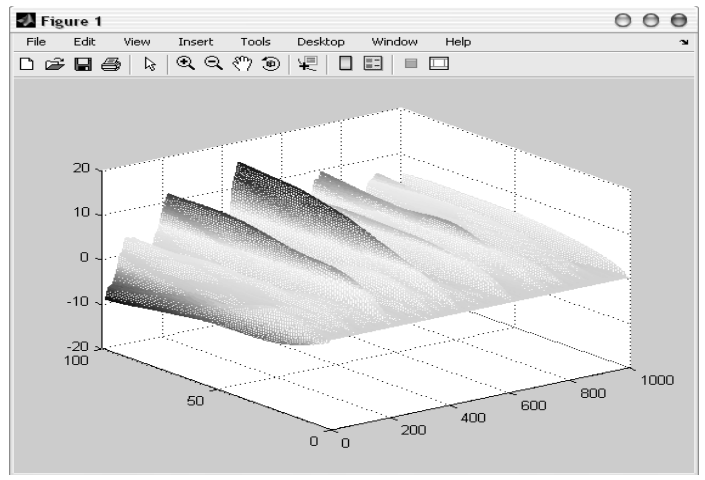

Figure 9. CWT of EGG for a subject with Bradygastria

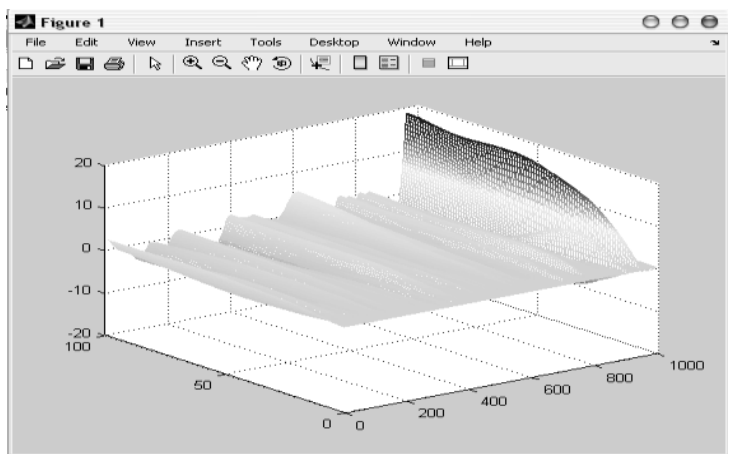

Figure 10. CWT of EGG for a subject with Tachygastria

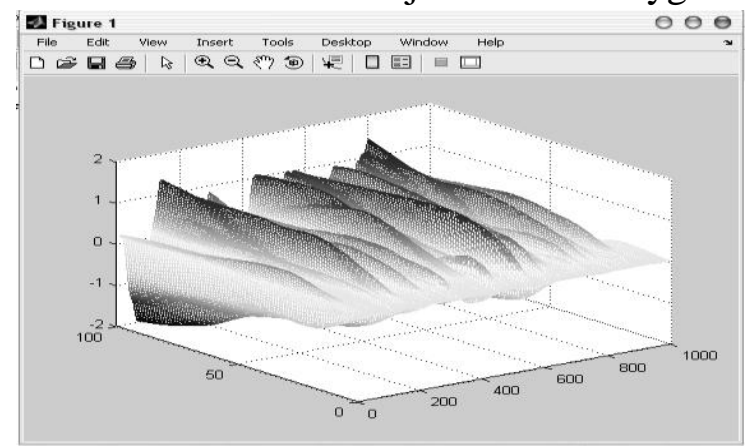


Figure 11.Bar graph representation Power variation in Patients with Dyspepsia (A) and Normal Individuals (B).

A

B

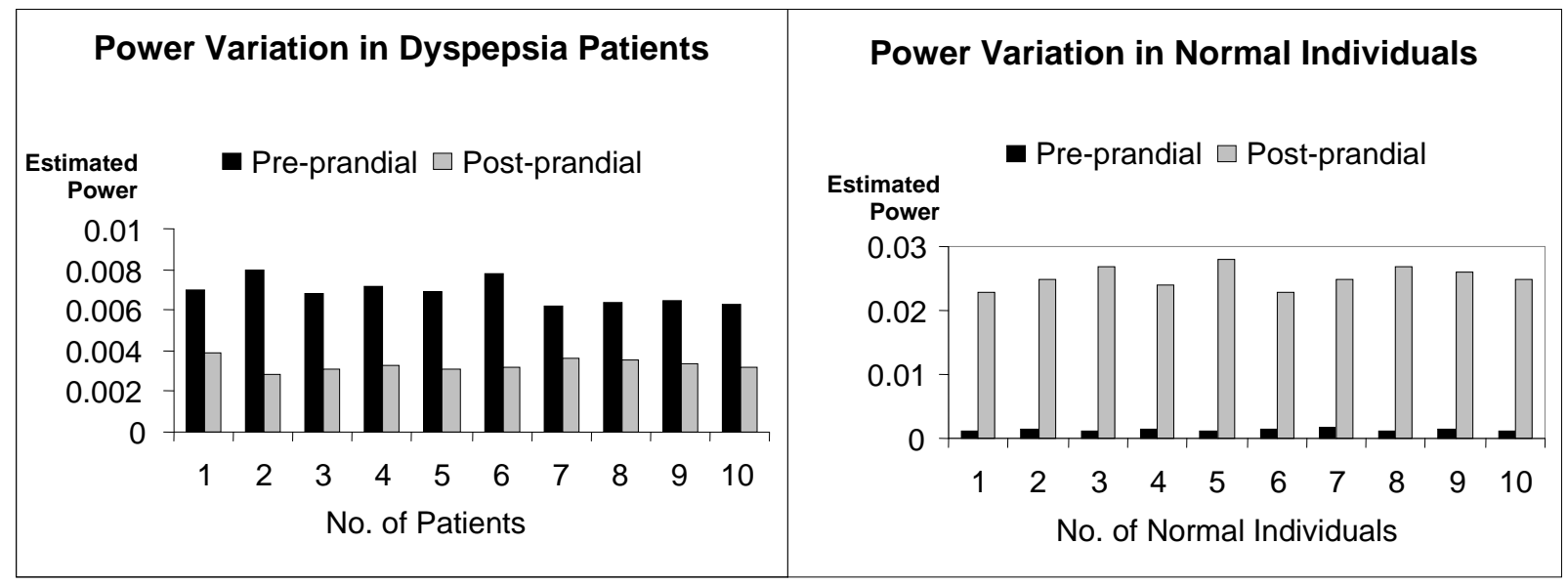

\section{Conclusions}

We have succeeded in acquiring the EGG ${ }^{(12,13)}$ from normal subjects and abnormal subjects who are suffering from different stomach disorders under monitoring and guidance of the gastroenterologist from a reputed hospital using active electrode and formed a database for EGG signals. The signal analysis is performed using MATLAB software in reconstructing, denosing, power and frequency calculation of the EGG signal from the database. The power and frequency of the EGG are compared and it is observed that when the power of the signal increased corresponding frequency is decreased for digestive disorder patient when compare to normal subjects. This fact may very much support the previous research work done in the field. The future work in this project would be to acquire EGG from patients suffering from different diseases to form a strong database with the hope that it would help digestive disorders more efficiently and precisely using EGG.

\section{Acknowledgements}

The authors acknowledge their indebtedness to the following medical experts $\operatorname{Dr} \mathrm{L}$ Venkatakrishnan, M.D., D.M.,D.N.B., Head of Gastroenterology Dept., Dr.J.Krishnaveni, M.D.,D.N.B., Gastroenterologist from PSG Hospitals, Coimbatore and Dr.T.S.Chandrasekar, M.D., D.M, Interventional Gastroenterologist for their support and for permitting us to use the facilities at the hospitals for live testing of the recording setup and sharing a valuable patient database with us. We are also acknowledging the Management, Principal, Director, Head of the BME/EIE of Sri Ramakrishna Engineering College, Coimbatore for their support in acquiring data from the different patients at the Department of Bio-Medical Engineering.

\section{References}

1. Alvarez WC. The Electrogastrogram and what it shows. JAMA 1922;78:1116-8.

2. Smout AJPM, Van Der Schee EJ, Grashuis JL. What is measured in electrogastrography? Digestive Diseases and Sciences 1980;25:179-187.

3. Code CF and Marlett JA. Modern Medical Physiology: Canine Tachygastria. Mayo Clinic Procedings 1974;49:325-32. 
4. Telander RL, Morgan KG, Kreulen DL, Schmalz PF, Kelly KA, et al. Human gastric atony with tachygastria and gastric retetion. Gastroenterology 1978;75:495-501.

5. Abell TL, Malagelada JR. Electrogastrography: Current assessment and future perspectives. Digestive Diseases and Sciences 1988;33:982-92.

6. Chen J, McCallum RW. Electrogastrography: measurement, analysis and prospective applications. Medical Biological Engineering and Computing 1991;29:339-50.

7. Riezzo G, Pezzolla F, Thouvenot J,Maselli MA, Giorgio I. Reproducibility of cutaneous recordings of electrogasography in the fasting state in man. Pathology Biology 1992;40:889-94.

8. Mintchev MP, Kingma YJ, Bowes KL. Accuracy of coutaneous recordings of gastric electrical activity. Gastroenterology 1993;104:1273-80.

9. Lin Z, Chen JDZ, Schirmer BD, McCallum RW. Postprandial response of gastric slow waves: correlation of serosal recording with the Electrogastrogram. Digestive Diseases and Sciences 2000;45(4):645-51.

10. Pfaffenban B, Adamek RJ, Kuhn K, Wegneer M. Electrogastrography in healthy subjects. Evalation of normal values influences of age and gender. Digestive Diseases and Sciences 1995;40:445-50.

11. Chen JDZ, Lin Z, Pan J, McCallum RW. Abnormal gastric myoelectrical activity and delayed gastric emptying in patients with symptoms suggestive of gastroparesis. Digestive Diseases and Sciences 1996;41:1538-45.

12. Chen JDZ. Non_invasive Measurement of gastric Myoelectrical Activity and its Analysis and Applications. Annual International Conference of the IEEE Engineering in Medicine and Biology Society 1998;20:2802-7.

13. Leahy A, Besherdas K, Clayman C, Mason I, Epstein O. Abnormalities of the electrogastrogram in functional dyspepsia. American Journal of Gastroenterology 1999;94:1023-28.

14. Han-Chang Wu, Kuang-Ching, Ya-Wen Chang; Full-Young Chang; Shuenn-Tsong Young, et al. Power distribution analysis of cutaneous Electrogastrography using discrete wavelet transform. International Conference of the IEEE Engineering in Medicine and Biology Society 1998;20:3227-29.

15. Chen DZ, Zhiyue Lin. Electrogastrogram-Encyclopedia of Medical Devices and Instrumentation, Second Edition, edited by John G. Webster. John Wiley. 2006.

16. Gopu G, Neelaveni R, Porkumaran K. Investigation of Digestive System Disorders using Electrogastrogram. ICCCE '08 proceeding international conference on Computer and Communication Engineering, 13-15 May 2008, Kuala Lumpur, Malaysia 2008;2:201-5.

17. Soman KP, Ramachandran KI. Insight to wavelets from Theory to Practice. Prentice Hall, New Delhi. 2004 